\title{
CRESCIMENTO DE MUDAS DE CANAFÍSTULA COM O USO DE ADUBAÇÃO BIOLÓGICA E BIOESTIMULANTE EM DIFERENTES SUBSTRATOS
}

\author{
Matheus Santin Padilha ${ }^{1}$, Carolina Riviera Duarte Maluche Baretta ${ }^{2}$, \\ Lúcia Salengue Sobral ${ }^{2}$, Elston_Kraft ${ }^{3}$, André Junior Ogliari ${ }^{4}$ \\ ${ }^{1}$ Mestrando do Programa de Pós-Graduação em Produção Vegetal, Universidade do \\ Estado de Santa Catarina (matheus_santin@hotmail.com) - Lages, SC - Brasil \\ ${ }^{2}$ Professora da Universidade Comunitária da Região de Chapecó - Chapecó, SC - \\ Brasil \\ ${ }^{3}$ Mestrando do Programa de Pós-Graduação em Ciência do Solo, Universidade do \\ Estado de Santa Catarina - Lages, SC - Brasil \\ ${ }^{4}$ Mestrando do Programa de Pós-Graduação em Ciências Ambientais, Universidade \\ Comunitária da Região de Chapecó - Chapecó, SC - Brasil
}

Recebido em: 06/04/2018 - Aprovado em: 10/06/2018 - Publicado em: 20/06/2018 DOI: 10.18677/EnciBio_2018A32

\begin{abstract}
RESUMO
O experimento teve como objetivo avaliar o crescimento de mudas de canafístula (Peltophorum dubium) produzidas com 0 uso de adubação biológica e bioestimulante em substrato orgânico e comercial. O experimento foi conduzido em delineamento experimental em blocos ao acaso, no arranjo fatorial $2 \times 4$, sendo dois substratos (orgânico e comercial), e quatro adubações com os fertilizantes testados (Testemunha, bioestimulante, fertilizante biológico, fertilizante biológico e bioestimulante), com quatro repetições. Os recipientes utilizados foram tubetes com $120 \mathrm{~cm}^{3}$ onde cada unidade experimental era composta por 30 mudas. Aos 100 dias após a semeadura as mudas foram avaliadas quanto aos seguintes parâmetros: altura da parte aérea $(\mathrm{H})$; diâmetro do coleto (DC); comprimento de raiz (CR); massa seca da parte aérea (MSPA); massa seca de raiz (MSR) e massa seca total (MST); relação da altura da parte aérea/diâmetro de coleto $(H / D C)$; relação entre altura da parte aérea/massa seca da parte aérea (H/MSPA); relação entre massa seca da parte aérea/massa seca de raiz (MSPA/MSR) e índice de qualidade de Dickson (IQD). O uso de fertilizante biológico e bioestimulante não apresentou resultado significativo na qualidade de mudas de Peltophorum dubium, e não apresentaram interação com os substratos testados. Os parâmetros avaliados H, DC, MSPA, MST e as relações H/DC e H/MSPA foram eficientes em indicar mudas de maior qualidade produzidas pelo substrato orgânico.
\end{abstract}

PALAVRAS-CHAVE: bioestimulante, Peltophorum dubium, substrato orgânico. 


\title{
GROWTH OF Peltophorum dubium (Spreng.) Taub SEEDLINGS WITH BIOLOGICAL FERTILIZER AND BIOSTIMULANT IN DIFERENT SUBSTRATES
}

\begin{abstract}
The objective of this study was evaluate the potential growth of Peltophorum dubium seedlings produced with biological fertilizer and biostimulant in organic compost and commercial substrate. The experiment has conducted in randomized blocks in a $2 \times 4$ factorial design, with two substrates (organic compost and commercial substrate) and four fertilizer (control, biostimulant, biological fertilizer, biostimulant plus biological fertilizer) with four replications. The was seedlings produced in tubes with $120 \mathrm{~cm}^{2}$ each plots has 30 plants. When seedling were at 100 days the following parameters were mensured: plant height, stem diameter, root lenght, shoot dry mass, dry mass of root, total dry mass, ratio between plant height and stem diameter, shoot dry mass, dry mass of root system, total dry mass, ratio between plant height and dry mass ratio of shoot, dry mass ratio of shoot/root dry mass and Dickson quality index. The biostimulant and biological fertilizer do not showed significant results as their use in the production of Peltophorum dubium seedlings. The parameters plant height, stem diameter, shoot dry mass, ratio between plant height and stem diameter and ratio between plant height and dry mass ratio of shoot were effective in indicating higher quality seedlings produced by the organic compost.
\end{abstract}

KEYWORDS: biostimulant, Peltophorum dubium, organic substrate.

\section{INTRODUÇÃO}

O Brasil é detentor da mais diversa flora do mundo, possuindo uma grande fonte natural de diversidade que pode ser utilizada para gerar ganhos econômicos, ambientais e sociais de forma satisfatória. Contudo, devido à ação antrópica sobre os ecossistemas naturais, identifica-se uma perda da biodiversidade dos ecossistemas (GONZAGA et al., 2016). Nesse contexto, a pesquisa deve atuar na geração de tecnologias e alternativas de manejo sustentáveis desses ecossistemas.

Estudos referentes às espécies que serão utilizadas na restauração ambiental e à forma como as mudas serão produzidas, são de extrema importância para garantir o sucesso da implantação e/ou revitalização desses ambientes (FARIA et al., 2016). A canafístula (Peltophorum dubium (Sprengel) Taubert) é uma espécie florestal nativa pertencente à família Fabaceae, sendo encontrada com frequência na Floresta Estacional Decidual. Além do seu alto potencial madeireiro devido à qualidade de sua madeira, a espécie destaca-se com grande potencial para uso em arborização urbana, recuperação de áreas degradadas e reflorestamento (CARVALHO, 2003).

No processo de produção de mudas, o substrato é um fator determinante para o desenvolvimento inicial, sendo que as características químicas, físicas e biológicas do mesmo influenciam diretamente no desempenho das espécies. Assim, para Siqueira et al. (2018), os substratos devem fornecer suporte físico às raízes e condições para suprir a demanda hídrica e nutricional das mudas.

Buscando a produção de mudas de forma mais sustentável, são encontrados no comércio diferentes tipos de fertilizantes orgânicos capazes de fornecer nutrientes, e alguns deles microrganismos benéficos que melhoram a qualidade do substrato. A utilização desses fertilizantes já é empregada na produção de mudas florestais e, trouxe resultados positivos na produção de mudas de Eucalyptus benthamii (MONTEIRO et al., 2017), Acacia mearnsii (HOPPE et al., 2004) e Ilex paraguariensis (HOPPE et al., 2005). 
Nessa perspectiva, o uso de bioestimulantes também vem sendo utilizado para a produção de mudas florestais e tem otimizado os processos fisiológicos de germinação e crescimento de mudas (PIEREZAN et al., 2012). Isso ocorre devido ao incremento hormonal e nutricional que os bioestimulante proporcionam (CARVALHO et al., 2013). Além disso, podem contribuir na melhoria das propriedades físicoquímicas do solo, na absorção, translocação e uso dos nutrientes pelas plantas, incluindo aumento de resistência a estresses abióticos, podendo ser usado nas diversas fases do desenvolvimento vegetal (RIBEIRO et al., 2017). Diante do exposto, este trabalho teve como objetivo avaliar o crescimento de mudas de canafístula (Peltophorum dubium) produzidas com o uso de adubação biológica e bioestimulante em substrato orgânico e comercial.

\section{MATERIAL E MÉTODOS \\ O experimento foi conduzido no Viveiro Florestal Universitário da} Universidade Comunitária da Região de Chapecó - Unochapecó, no município

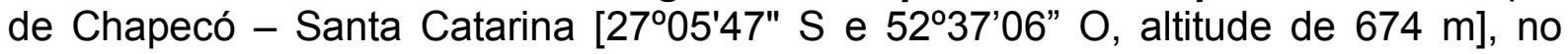
período de março a novembro de 2015.

As sementes de Peltophorum dubium foram provenientes de oito árvores matrizes localizadas em quatro fragmentos florestais do Distrito de Marechal Bormann, localizado no mesmo município. Após a coleta os frutos foram levados ao Laboratório de Sementes, da Área de Ciências Exatas e Ambientais da Unochapecó, onde a abertura dos mesmos foi realizada de forma manual seguido do beneficiamento das sementes para eliminação das impurezas e sementes danificadas.

O delineamento experimental foi em blocos ao acaso com quatro repetições, em arranjo fatorial $2 \times 4$, com dois substratos e quatro adubações testadas, totalizando 32 unidades experimentais. Cada unidade experimental foi composta por 30 mudas, sendo que as 12 mudas centrais corresponderam à área útil.

Os substratos avaliados foram um substrato orgânico produzido na unidade de compostagem do Viveiro Florestal Universitário a partir de resíduos vegetais das áreas verdes e restaurantes do campus e, um substrato comercial formulado a base de turfa, vermiculita expandida e casca de arroz carbonizada. Os substratos foram peneirados em peneira com malha de $4 \mathrm{~mm}$ de diâmetro e caracterizados quimicamente no Laboratório de Análise de Solos da Empresa de Pesquisa Agropecuária e Extensão Rural de Santa Catarina (EPAGRI/Cepaf/Chapecó), apresentando as seguintes características: $38,47 \%$ de umidade, $\mathrm{pH}_{\mathrm{H} 2 \mathrm{O}}=7,2, \mathrm{~N}=6,7$ $\mathrm{g} \mathrm{dm}^{3}, \mathrm{P}=3,6 \mathrm{~g} \mathrm{dm}^{3}, \mathrm{~K}=1,8 \mathrm{~g} \mathrm{dm}^{3}, \mathrm{Ca}=2,4 \mathrm{~g} \mathrm{dm}^{3}, \mathrm{Mg}=1,5 \mathrm{~g} \mathrm{dm}^{3}, \mathrm{Cu}=0,002 \mathrm{mg}$ $\mathrm{dm}^{3}, \mathrm{Zn}=0,06 \mathrm{mg} \mathrm{dm}{ }^{3}, \mathrm{Fe}=28,48 \mathrm{mg} \mathrm{dm}^{3}, \mathrm{Mn}=0,12 \mathrm{mg} \mathrm{dm}^{3}$ (substrato orgânico) e; $43,43 \%$ de umidade, $\mathrm{pH}_{\mathrm{H} 2 \mathrm{O}}=5,7, \mathrm{~N}=3,2 \mathrm{~g} \mathrm{dm}^{3}, \mathrm{P}=2,8 \mathrm{~g} \mathrm{dm}^{3}, \mathrm{~K}=3,0 \mathrm{~g} \mathrm{dm}^{3}, \mathrm{Ca}=$ $4,1 \mathrm{~g} \mathrm{dm}^{3}, \mathrm{Mg}=2,1 \mathrm{~g} \mathrm{dm}^{3}$ (substrato comercial).

As adubações testadas constituíram-se de um fertilizante biológico $100 \%$ natural (BacSolß) composto de bactérias dos gêneros Bacillus, Pseudomonas, Nitrosomonas e Nitrobacter (MONTEIRO et al., 2014), e fungos como Saccharomyces cerevisiae (SPANIOL et al., 2014) e, um fertilizante orgânico

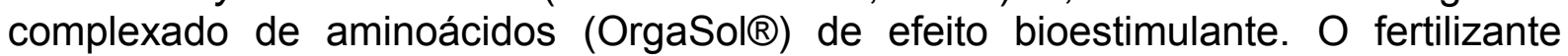
biológico (BacSol尺) apresenta em sua composição $\mathrm{N}$ total $=5 \%, \mathrm{pH}=6,0$; carbono orgânico = 45\%; CTC = $405 \mathrm{mmol} \mathrm{dm}$; CTC/C = 10 enquanto; o bioestimulante (OrgaSol囚) é composto por S = 3,5\%; $B=0,5 \% ; M n=2,5 \% ; M o=0,01 \%$ e $\mathrm{Zn}=4 \%$ (RSA INDÚSTRIA DE INSUMOS AGRÍCOLA LTDA, 2014). 
Antes da semeadura as sementes de Peltophorum dubium foram imersas em água a $80^{\circ} \mathrm{C}$ por cinco minutos para a superação da dormência e, na sequência,

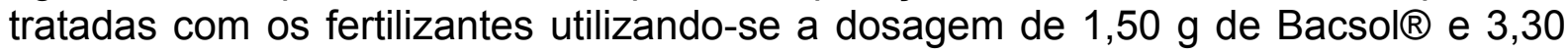
$\mathrm{ml}$ de Orgasol ${ }^{\circ}$ por quilo de semente, valores adaptados de Hoppe et al. (2004).

Dessa forma os tratamentos testados foram assim identificados: substrato orgânico/sementes sem tratamento (T1); substrato orgânico/sementes tratadas com fertilizante biológico (T2); substrato orgânico/sementes tratadas com bioestimulante (T3); substrato orgânico/sementes tratadas com fertilizante biológico e bioestimulante (T4); substrato comercial/sementes sem tratamento (T5); substrato comercial/sementes tratadas com fertilizante orgânico (T6); substrato comercial/sementes tratadas com bioestimulante (T7); substrato comercial/sementes tratadas com fertilizante biológico e bioestimulante (T8).

A semeadura foi realizada utilizando três sementes em cada tubete cilíndrico com $120 \mathrm{~cm}^{3}$ de capacidade, dispostos em bancadas com 1,0 m de altura, sendo que na instalação do experimento os substratos foram umedecidos de forma a manterem um teor de água equivalente a $80 \%$ da sua capacidade de campo. As mudas eram irrigadas duas vezes ao dia, no início da manhã e ao final da tarde, durante aproximadamente 20 minutos, pelo sistema de aspersão da casa de vegetação. Aos 40 dias da semeadura foi realizado o desbaste, deixando-se apenas uma plântula por tubete.

Aos 100 dias após a semeadura as mudas de Peltophorum dubium foram avaliadas quanto à altura da parte aérea $(\mathrm{H})$ medida com uma régua graduada em milímetros a partir da base da planta até o meristema apical; comprimento de raiz (CR) determinado com o uma régua graduada em milímetros a partir do colo até a extremidade da raiz mais longa; diâmetro de coleto (DC) determinado na base do caule da planta com o uso de um paquímetro digital Vonder Modelo Pd 150. Foram ainda avaliadas à massa seca da parte aérea (MSPA) e massa seca de raiz (MSR), através de secagem em estufa com circulação forçada de ar a $65^{\circ} \mathrm{C}$ até peso constante, e pesagem realizada em balança analítica com precisão de 0,0001g, e calculada à massa seca total (MST) pelo somatório da MSPA e MSR.

A partir dos dados obtidos foram determinadas as relações de altura/diâmetro de coleto (H/DC), altura/massa seca de parte aérea (H/MSPA), massa seca de parte aérea/ massa seca de raiz (MSPA/MSR), e por fim foi determinado o índice de qualidade de Dickson [IQD = MST/(H/D + MSPA/MSR)] (DICKSON et al., 1960). Os resultados obtidos foram submetidos à análise de variância pelo teste $\mathrm{F}(p<0,05)$, e a comparação entre as médias e interações efetuada pelo teste de Tukey a $5 \%$ de probabilidade.

\section{RESULTADOS E DISCUSSÃO}

Para todos os parâmetros avaliados o teste $F$ não demonstrou efeito significativo para a interação entre os fatores (substratos e fertilizantes), assim como não foi verificado efeito significativo isolado da utilização do fertilizante biológico

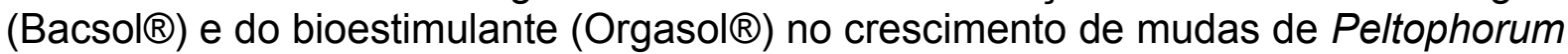
dubium. A análise de variância indicou diferença significativa apenas para o fator substrato com relação ao comprimento de raiz (CR), diâmetro de coleto (DC), altura de parte aérea $(H)$, massa seca da parte aérea (MSPA), massa seca total (MST), e para as relações H/DC, H/MSPA e MSPA/MSR (Tabela 1).

Para Peltophorum dubium observou-se que tanto o Bacsol $₫$ como o Orgasol ( não promoveram resultados positivos na produção das mudas, até os 100 dias após a semeadura indicando que a espécie pode não ser responsiva aos produtos 
utilizados. Corroborando com os resultados obtidos, Hoppe et al. (2004) verificaram que a aplicação do bioestimulante Orgasol $\circledast$ no tratamento de sementes não afetou a qualidade das mudas de Acacia mearnsii. No entanto, os mesmos autores verificaram que a aplicação do fertilizante Bacsolß nas sementes de Acacia mearnsii demonstrou resultados positivos aos 90 dias após a semeadura, sendo que para a variável $\mathrm{H}$, o incremento foi de 57 a $96 \%$ superior a testemunha, o mesmo evidenciado para a variável DC onde foi de 25 a $33 \%$ superior, indicando diferença na resposta dos produtos para cada espécie.

TABELA 1 - Valores de quadrado médio (QM) referentes à produção de mudas de Peltophorum dubium aos 100 dias após semeadura.

\begin{tabular}{ccccccc}
\hline \multirow{2}{*}{ FV } & GL & \multicolumn{5}{c}{ QM } \\
\cline { 3 - 7 } & & $\mathbf{H}$ & $\mathbf{D C}$ & $\mathbf{C R}$ & MSPA & MSR \\
\hline Bloco & 3 & 0,46 & 0,04 & 1,44 & 0,01 & 0,001 \\
Fertilizante (F) & 3 & $0,88^{\text {ns }}$ & $0,04^{\text {ns }}$ & $1,07^{\text {ns }}$ & $0,01^{\text {ns }}$ & $0,001^{\text {ns }}$ \\
Substrato (S) & 1 & $323,02^{* *}$ & $3,64^{* *}$ & $106,78^{* *}$ & $0,94^{* *}$ & $0,000^{\text {ns }}$ \\
Fx S & 3 & $0,66^{\text {ns }}$ & $0,03^{\text {ns }}$ & $1,99^{\text {ns }}$ & $0,01^{\text {ns }}$ & $0,000^{\text {ns }}$ \\
\hline & & MST & H/DC & H/MSPA & MSPA/MSR & IQD \\
\hline Bloco & 3 & 0,004 & 0,064 & 10,53 & 1,89 & 1,47 \\
Fertilizante (F) & 3 & $0,003^{\text {ns }}$ & $0,160^{\text {ns }}$ & $0,86^{\text {ns }}$ & $0,95^{\text {ns }}$ & $0,38^{\text {ns }}$ \\
Substrato (S) & 1 & $0,959^{* *}$ & $14,137^{* *}$ & $323,45^{* *}$ & $82,79^{* *}$ & $1,68^{\text {ns }}$ \\
Fx S & 3 & $0,009^{\text {ns }}$ & $0,125^{\text {ns }}$ & $17,66^{\text {ns }}$ & $0,40^{\text {ns }}$ & $0,19^{\text {ns }}$ \\
\hline
\end{tabular}

Legenda: ${ }^{\text {n }}$ valor de $\mathrm{F}$ não significativo a $1 \%$; ** valor significativo a $1 \%$ de probabilidade; $F V=$ fonte de variação; $G L=$ grau de liberdade. Fertilizante $(F)$ : Bacsol $\circledast$ e Orgasol@ . Substratos $(S)$ : substrato orgânico e substrato comercial; Altura de planta (H); diâmetro de coleto (DC); comprimento de raiz (CR); massa seca de parte aérea (MSPA); massa seca de raiz (MSR); massa seca total (MST); relação altura/diâmetro de coleto (H/DC); relação altura/massa seca de parte aérea (H/MSPA); relação massa seca de parte aérea/massa seca de raiz (MSPA/MSR) e indice de qualidade de Dickson (IQD).

A avaliação do efeito de bioestimulantes foi estudada também por Pierezan et al. (2012) onde os autores não verificaram diferença significativa do uso do bioestimulante Stimulate $₫$ no crescimento e nem no metabolismo das mudas de Hymenaea courbaril aos 100 dias de avaliação. O mesmo foi relatado por Missio e Moro (2016) avaliando o uso do bioestimulante Stimulate ${ }^{\circledR}$ na produção de mudas de Parapiptadenia rigida. Contudo, os autores acima destacam que são necessários mais estudos sobre as doses utilizadas dos produtos para o tratamento de sementes.

O fertilizante Bacsol® foi utilizado misturado ao substrato por Monteiro et al. (2017), e proporcionou resultados positivos com a aplicação de doses de 0,5; 1,0; 1,5 e 2,0 g do produto/muda na produção de Eucalyptus benthamii aos 120 dias, sob as variáveis $\mathrm{H}$, MSPA e relação $\mathrm{H} / \mathrm{MSPA}$. Ainda no mesmo estudo, o tratamento com Bacsol $\circledast$ promoveu para a variável $\mathrm{H}$ aumento no crescimento de 40 a $76 \%$ em relação à testemunha, assim como a MSPA com pesos de 89 a $169 \%$ superiores ao controle, e relação H/MSPA com índices de 28 a $41 \%$ inferiores ao controle.

Utilizando a aplicação no substrato Hoppe et al. (2005) verificaram que doses de 600 a $800 \mathrm{~g}$ de Bacsol $8 / \mathrm{m}^{3}$ de substrato promoveram um aumento de 90 a $168 \%$ na variável $\mathrm{H}$ e de 41 a $52 \%$ na variável DC em mudas de Ilex paraguariensis com 100 dias, demonstrando ser um produto promissor para o uso na produção de mudas. 
O uso de diferentes tecnologias para produção de mudas é uma alternativa para aperfeiçoar a produção, sendo que Monteiro et al. (2014) ressaltam que produtos biotecnológicos apresentam potencial de utilização para a Silvicultura aumentando a qualidade das mudas florestais e diminuindo os custos de produção, quantidade de fertilizantes utilizados, ciclo de produção e incidência de pragas e doenças. Os autores citam o Bacsol®, bem como outros produtos que apresentam na sua composição microrganismos benéficos onde, através do estudo para algumas espécies florestais, tem demonstrado efeito positivo na promoção do crescimento dessas plantas e melhoria da qualidade das mudas.

No entanto, embora a recomendação técnica indique os produtos testados para o tratamento de sementes, é necessário aprimoramento em relação aos métodos de aplicação e doses utilizadas, assim como, o estudo em outras espécies; uma vez que, muitas espécies florestais possuem simbiose com microrganismos benéficos que podem trazer ganhos econômicos e ecológicos para o setor florestal (BARBERI et al., 1998).

Com relação aos substratos a Tabela 2 demonstra os resultados obtidos para $\mathrm{H}$, CR e DC. Observa-se para o parâmetro $\mathrm{H}$ que o melhor resultado foi observado para o substrato orgânico, o qual promoveu um incremento na altura da parte aérea em relação ao substrato comercial.

TABELA 1 - Médias da altura de parte aérea $(H)$, comprimento de raiz $(C R)$ e diâmetro de coleto (DC) das mudas de Peltophorum dubium produzidas em substrato orgânico e comercial.

\begin{tabular}{cccr}
\hline Substrato & $\mathbf{H}$ & $\begin{array}{c}\text { CR } \\
---\mathbf{c m} \text {--- }\end{array}$ & DC \\
\hline Orgânico & $14,33 \pm 1,20 \mathrm{a}^{*}$ & $19,69 \pm 0,43 \mathrm{~b}$ & $2,98 \pm 0,15 \mathrm{a}$ \\
Comercial & $7,97 \pm 0,84 \mathrm{~b}$ & $23,35 \pm 1,94 \mathrm{a}$ & $2,31 \pm 0,20 \mathrm{~b}$ \\
\hline CV $(\%)$ & 10,5 & 5,71 & 6,51 \\
\hline
\end{tabular}

${ }^{*}$ Médias com a mesma letra não diferem estatisticamente $(p<0,05)$ pelo teste de Tukey.

Corroborando os resultados obtidos no presente estudo Rodrigues et al. (2016), verificaram que mudas de Moringa oleifera apresentaram maior crescimento de plantas quando produzidas em substrato orgânico, e ressaltam que o composto oriundo da compostagem apresenta condições favoráveis ao crescimento das plantas, devido a boa aeração, estrutura e, principalmente, pelo fornecimento de nutrientes prontamente disponíveis.

A utilização de um substrato com sua composição baseada em $100 \%$ de substrato orgânico, promoveu maior crescimento das mudas de Peltophorum dubium, divergindo dos resultados encontrados por Caldeira et al. (2008) que, ao estudarem a produção de mudas de Schinus terebinthifolius, verificaram que a utilização de um substrato constituído por $100 \%$ de composto orgânico oriundo de compostagem foi prejudicial ao crescimento da parte aérea das mudas desta espécie, o que indica que a concentração de composto orgânico em um substrato pode apresentar resposta diferente de acordo com a espécie e as suas necessidades.

O diâmetro de coleto (DC) é um parâmetro de fácil avaliação, não destrutivo e que pode ser utilizado para indicar a qualidade das mudas. Os melhores resultados de DC foram observados nas mudas produzidas com substrato orgânico, sendo que promoveu um aumento de $22,80 \%$ no DC em relação aos valores obtidos para esta variável no substrato comercial. 
Vieira et al. (2014) destacam que o DC de mudas de Calophyllum brasiliense foram influenciadas pela adição de fontes orgânicas na formulação do substrato, e acrescentam que isso ocorre devido a fontes orgânicas aumentarem a fertilidade do mesmo. Resultados obtidos por Cruz et al. (2011), avaliando o efeito de doses de macronutrientes na produção de mudas de Peltophorum dubium constataram que as mudas respondem a aplicação de macronutrientes para todas as variáveis analisadas, inclusive o DC. A partir disso, o substrato orgânico por apresentar maior concentração de nutrientes em relação ao substrato comercial, promoveu o maior DC.

Com relação ao CR observa-se que o substrato comercial promoveu o maior CR quando comparado ao substrato orgânico, sendo este valor $17,64 \%$ superior. Segundo Steffen et al. (2011), esse resultado pode ser explicado pelo fato de substratos constituídos por $100 \%$ de composto orgânico originado de compostagem afetarem negativamente o crescimento radicular devido ao maior adensamento das partículas, quando comparados a outros tratamentos os quais foram utilizados turfa na mistura do substrato, por exemplo, fato evidenciado em sua pesquisa com produção de mudas de Corymbia citriodora.

Neste sentido, é importante salientar que um sistema radicular com maior comprimento promove maior absorção de água e nutrientes, já que alcança maior profundidade no perfil do solo, sendo tal característica influenciada pela disponibilidade de água, nutrientes, resistência à penetração e aeração do substrato (SALTON; TOMAZI, 2014).

A Tabela 3 demonstra os resultados obtidos para MSPA, MSR e MST. Para MSPA e MST os maiores valores encontrados foram obtidos com o uso do substrato orgânico (Tabela 3), que também apresentou os maiores valores $H$ (Tabela 2), contudo a MSR não demonstrou diferença entre os substratos utilizados. A massa seca de parte aérea revela que as mudas produzidas em substrato orgânico apresentavam maior capacidade fotossintética o que beneficia o desenvolvimento das mudas e a sua sobrevivência no campo após a expedição.

TABELA 2 - Médias de massa seca de parte aérea (MSPA), massa seca de raiz (MSR) e massa seca total (MST) de mudas de Peltophorum dubium produzidas em substrato orgânico e comercial.

\begin{tabular}{cccc}
\hline Substrato & MSPA (g) & MSR (g) & MST (g) \\
\hline Orgânico & $0,613 \pm 0,07 \mathrm{a}^{*}$ & $0,109 \pm 0,02$ a & $0,722 \pm 0,07 \mathrm{a}$ \\
Comercial & $0,268 \pm 0,05 \mathrm{~b}$ & $0,107 \pm 0,04 \mathrm{a}$ & $0,375 \pm 0,09 \mathrm{~b}$ \\
\hline CV $(\%)$ & 14,60 & 25,94 & 14,66 \\
\hline
\end{tabular}

"Médias com a mesma letra não diferem estatisticamente $(p<0,05)$ pelo teste de Tukey.

Toledo et al. (2015) constataram que a formulação de substratos orgânicos através do uso de resíduos orgânicos na produção de mudas de Eucaliptus grandis, promoveram o incremento de MSPA. Ao contrário, Vieira et al. (2014) verificaram que a utilização de resíduos orgânicos independente da mistura utilizada não influenciou na variável MSPA de mudas de Calophyllum brasiliense.

Mesmo que o CR apresente menor comprimento no substrato orgânico (Tabela 2) as mudas produzidas manifestaram maior valor de MSPA. Segundo Steffen et al. (2011), tal característica está relacionada ao direcionamento de assimilados para o maior crescimento em altura pelas mudas que se desenvolvem em substrato mais rico em nutrientes. Concomitantemente, apesar de existir diferenças entre o $\mathrm{CR}$ das mudas em cada substrato, não houve diferença significativa entre a MSR. 
A média da MST das mudas produzidas no substrato orgânico foi $92 \%$ superior à massa seca das mudas do substrato comercial. Corroborando os resultados obtidos, Faria et al. (2013) constataram que substratos com $80 \%$ de resíduo orgânico promoveram melhores resultados quanto a MST na produção de mudas de Senna alata. Dessa forma, Mussi et al. (2013) consideram que é essencial que os substratos possuam uma concentração de resíduo orgânico em quantidades adequadas para as necessidades de cada espécie, e verificaram que para mudas de Peltophorum dubium o substrato deve possuir em sua composição uma fonte orgânica para que ocorram ganhos de MSPA, MSR e MST.

A obtenção das relações entre os parâmetros morfológicos são combinações de valores que buscam expressar mais precisamente o potencial de sobrevivência de uma muda a campo. Para as relações H/DC, H/MSPA e MSPA/MSR, os melhores valores encontrados foram para as mudas produzidas no substrato orgânico, entretanto, O IQD não apresentou diferença significativa entre os substratos (Tabela 4).

TABELA 3 - Médias das relações altura de planta/diâmetro de coleto (H/DC), altura de planta/massa seca de parte aérea (H/MSPA) e massa seca de parte aérea/massa seca de raiz (MSPA/MSR) das mudas de Peltophorum dubium produzidas em substrato orgânico e comercial.

\begin{tabular}{ccccc}
\hline Substrato & H/DC & H/MSPA & MSPA/MSR & IQD \\
\hline Orgânico & $4,79 \pm 0,57 \mathrm{a}^{*}$ & $23,58 \pm 2,17 \mathrm{~b}$ & $5,85 \pm 1,31 \mathrm{a}$ & $0,83 \pm 0,15 \mathrm{a}$ \\
Comercial & $3,46 \pm 0,06 \mathrm{~b}$ & $30,23 \pm 3,09 \mathrm{a}$ & $2,63 \pm 0,59 \mathrm{~b}$ & $0,75 \pm 0,23 \mathrm{a}$ \\
\hline CV $(\%)$ & 10,52 & 13,65 & 25,91 & 21,94 \\
\hline
\end{tabular}

*Médias com a mesma letra não diferem estatisticamente $(p<0,05)$ pelo teste de Tukey.

Aos 100 dias após a semeadura, os valores da relação H/DC foram de 4,79 para o substrato orgânico e 3,46 para o comercial, corroborando os resultados obtidos por Cruz et al. (2011), que verificaram que mudas de Peltophorum dubium com 120 dias produzidas em Latossolo Vermelho-Amarelo distrófico, apresentaram relação H/DC entre 2,89 a 4,61. Entretanto, o substrato orgânico demonstrou relação de H/DC superior ao substrato comercial, fato também verificado por Mussi et al. (2013) e, verificaram que o uso de fontes orgânicas na produção das mudas de Peltophorum dubium proporcionou valores da relação H/DC entre 4,46 e 6,12 aos 180 dias após a semeadura.

Conforme resultados obtidos por Souza et al. (2017), as mudas com maiores valores da relação H/DC apresentam um maior equilíbrio no seu desenvolvimento, sendo mais robustas. Quando a relação H/DC for muito elevada às mudas devem ficar mais tempo no viveiro para reduzir o desequilíbrio entre essas variáveis e garantir um maior sucesso do transplante para o campo.

A relação H/MSPA segundo Gomes e Paiva (2004), demonstra que quanto menor o valor do índice maior é chance de sobrevivência da muda no campo, sendo um indicativo do quanto estão lignificadas as plantas. O substrato orgânico proporcionou a formação de mudas de Peltophorum dubium com uma relação H/MSPA de 23,58, a qual foi significativamente inferior à obtida no substrato comercial, cujo valor foi de 30,23 , indicando melhor qualidade das mudas no substrato orgânico. Trabalhando com a mesma espécie Cruz et al. (2012), obtiveram resultados semelhantes, sendo que ao avaliar doses de macronutrientes na produção das mudas, verificaram que as mudas que não receberam adubação com macronutrientes apresentaram valores de H/MSPA de 26,22 aos 120 dias. 
As mudas produzidas no substrato orgânico demonstraram relação MSPA/MSR de 5,85 se comparadas ao comercial o qual foi de 2,63. Caldeira et al. (2013) obtiveram valores de MSPA/MSR para mudas de Chamaecrista desvauxii entre 2,52 e 3,77 , e verificaram que o substrato comercial utilizado promoveu o menor valor desse índice sendo 0,92 , dados semelhantes aos obtidos pela presente pesquisa. Contudo, Caldeira et al. (2008) ressaltam que para mudas mais equilibradas a relação ideal para esse índice deve ser 2,0 e, complementam que a parte aérea não deve ser muito superior a raiz, pois podem ocorrer problemas na adaptação das mudas em relação a absorção de água e nutrientes. A partir disso, o substrato comercial obteve resultado mais próximo do adequado para uma muda quanto à relação MSPA/MSR.

\section{CONCLUSÃO}

O uso de adubação biológica e bioestimulantes nas condições testadas não influenciaram o crescimento e desenvolvimento de mudas de Peltophorum dubium.

O substrato orgânico proveniente de compostagem proporcionou a produção de mudas de melhor qualidade em relação ao substrato comercial, sendo uma opção viável na produção de mudas de espécies florestais.

\section{AGRADECIMENTOS}

Os autores agradecem ao Viveiro Florestal Universitário pela disponibilidade do espaço e equipamentos para execução da pesquisa.

\section{REFERÊNCIAS}

BARBERI, A.; MARCO A. C. CARNEIRO, M. A. C.; FÁTIMA M. S. MOREIRA, F. M. S.; SIQUEIRA, J. O. Nodulação em leguminosas florestais em viveiros no sul de minas gerais. Cerne, v. 4, n. 1, p. 145-153, 1998. Disponível em:

<http://www.cerne.ufla.br/site/index.php/CERNE/article/download/611/520>.

CALDEIRA, M. V. W.; DELARMELINA, W. M.; FARIA, J. C. T.; JUVANHOL, R. S. Substratos alternativos na produção de mudas de Chamaecrista desvauxii. Revista Árvore, v. 37, n. 1, p. 31-39, 2013. Disponível em: <http://dx.doi.org/10.1590/S0100-67622013000100004>.doi:10.1590/S010067622013000100004

CALDEIRA, M. V. W.; ROSA, G. N.; FENILLI, T. A. B.; HARBS, R. M. P. Composto orgânico na produção de mudas de Aroeira-Vermelha. Scientia Agrária, v. $9, \quad$ n. $1, \quad$ p. $27-33, \quad 2008$. Disponível em: <http://dx.doi.org/10.5380/rsa.v9i1.9898>. doi: 10.5380/rsa.v9i1.9898

CARVALHO, P. E. R. Espécies arbóreas brasileiras. Brasília, DF: Embrapa Informação Tecnológica, v. 1, 2003. 1039 p.

CARVALHO, T. C. de.; SILVA, S. S. da.; SILVA, R. C. da.; PANOBIANCO, M.; MÓGOR, A. F. Influência de bioestimulantes na germinação e desenvolvimento de plântulas de Phaseolus vulgaris sob restrição hídrica. Revista de Ciências Agrárias, v. $36, \quad$ n. 2 , p. 199-205, 2013. Disponível em: $<$ http://www.scielo.mec.pt/scielo.php?script=sci_abstract\&pid=S0871018X2013000200009>. 
CRUZ, C. A. F.; PAIVA, H. N.; CUNHA, A. C. M. C. M.; NEVES, J. C. L. Produção de mudas de canafístula cultivadas em Latossolo Vermelho amarelo álico em resposta a macronutrientes. Cerne, v. 18, n. 1, p. 87-98, 2012. Disponível em: <http://dx.doi.org/10.1590/S0104-77602012000100011>. doi:10.1590/S010477602012000100011

CRUZ, C. A. F.; PAIVA, H. N.; MORI da CUNHA, A. C. M. C.; NEVES, J. C. L. Macronutrientes na produção de mudas de canafístula em Argissolo Vermelho amarelo da região da Zona da Mata, MG. Ciência Florestal, v. 21, n. 3, p. 445-457, 2011. Disponível em: <http://dx.doi.org/10.5902/198050983802>. doi: $10.5902 / 198050983802$

DICKSON, A.; LEAF, A. L.; HOSNER, J. F. Quality appraisal of while spruce and white pine seedling stock in nurseries. Forestry Chronicle, v. 36, p. 11-13, 1960. Disponível em: < https://doi.org/10.5558/tfc36010-1>. doi: 10.5558/tfc36010-1

FARIA, J. C. T.; CALDEIRA, M. V. W.; DELARMELINA, W. M.; LACERDA, L. C.; GONÇALVES, E. O. Substratos à base de lodo de esgoto na produção de mudas de Senna alata. Comunicata Scientiae, v. 4, n. 4, p. 342-351, 2013. Disponível em: <https://dialnet.unirioja.es/descarga/articulo/5022019.pdf>

FARIA, J. C. T.; CALDEIRA, M. V. W.; DELARMELINA, W. M.; ROCHA, R. L. F. Substratos alternativos na produção de mudas de Mimosa setosa benth. Ciência Florestal, v. 26, n. 4, p. 1075-1086, 2016. Disponível em: <http://dx.doi.org/10.5902/1980509824996>. doi: 10.5902/1980509824996

GOMES, J. M; PAIVA, H. N. Viveiros florestais. Propagação sexuada. Viçosa: UFV, 2004. 116 p.

GONZAGA, L. M.; SILVA, S. S.; CAMPOS, S. A.; FERREIRA, R. P.; CAMPOS, A. N. R. et al. Recipientes e substratos para a produção de mudas de jatobá (Hymenaea courbaril L.). Revista Brasileira de Agropecuária Sustentável, v. 6, n. 1, p. 64-73, 2016. Disponível em: <http://dx.doi.org/10.21206/rbas.v6i1.309>. doi: 10.21206/rbas.v6i1.309

HOPPE, J. M.; SCHUMACHER , A. V.; QUEVEDO, F. F.; THOMAS, R.; IVANOV, G. et al. Uso do Bacsol ${ }^{\circledR}$ na produção de mudas de erva-mate (Ilex paraguariensis). In: Relatório de pesquisa: uso do Bacsol $($ em diferentes pesquisas. Santa Maria, RS: Universidade Federal de Santa Maria, 2005. p. 66-78. Disponível em: <http://www.rsa.ind.br/midia/ rel_pesq.pdf>.

HOPPE, J. M.; SCHUMACHER , A. V.; QUEVEDO, F. F.; CÍCERO, G.; THOMAS, R. et al. Aplicação de diferentes doses de Bacsol $\cap$ e Orgasol $\circledast$ em sementes de acácia-negra (Acacia mearnsii D. Willd.) e seu desenvolvimento no viveiro. In: Santa Maria, RS: Universidade Federal de Santa Maria, 2004. p. 43-56. Disponível em: <http://rsa.ind.br/midia/Bacsol Orgasol-UFSM.pdf>.

MISSIO, E. L.; MORO, T. Tratamento de sementes na produção de mudas de angico vermelho. Caderno de Pesquisa, v. 28, n. 2, p. 38-51, 2016. Disponível em: <http://dx.doi.org/10.17058/cp.v28i2.7127 >. doi: 10.17058/cp.v28i2.7127 
MONTEIRO, P. H. R.; WINAGRASKI, E.; AUER, C. G. Importância do uso de rizobactérias na produção de mudas florestais. Colombo: Embrapa Florestas, 2014. 6 p. (Embrapa Florestas. Comunicado técnico, 338).

MONTEIRO, P. E. R.; WINAGRASKI, E.; KASCHUK, G.; GAIAD, S.; MARQUES, R.; AUER, C. G. Responses of Eucalyptus benthamii seedlings to the application of the organic fertilizer Bacsol. Bosque, v. 38, n. 3, p. 507-513, 2017. Disponível em: https://www.embrapa.br/florestas/busca-depublicacoes/-/publicacao/1082579/responses-of-eucalyptus-benthamiiseedlings-to-the-application-of-the-organic-fertilizer-bacsol. doi: 10.4067/S0717-92002017000300008.

MUSSI, N. S.; CARVALHO, M. O.; SILVA, M. M.; CAMPOS, A. N. R.; CUNHA, A. C. M. C. M. Substratos orgânicos na produção de mudas de canafístula. Cadernos de Agroecologia, v. 8, n. 2, p. 1-5, 2013. Disponível em: <http://revistas.abaagroecologia.org.br/index.php/cad/article/view/14209>.

PIEREZAN, L.; SCALON, S. P. Q.; PEREIRA, Z. V. Emergência de plântulas e crescimento de mudas de jatobá com uso de bioestimulante e sombreamento. Cerne, v. 18, n. 1, p. 127-133, 2012. Disponível em: <http://dx.doi.org/10.1590/S0104-77602012000100015>. doi: 10.1590/S010477602012000100015.

RIBEIRO, R. F.; LOBO, J. T.; CAVALCANTE, Í. H. L.; TENREIRO, I. G. P.; LIMA, D. D. Bioestimulante na produção de mudas de videira $\mathrm{cV}$. Crimson seedless. Scientia Agrária, v. 18, n. 4, p. 36-42, 2017. Disponível em: <http://dx.doi.org/10.5380/rsa.v18i4.50922>. doi: 10.5380/rsa.v18i4.50922

RODRIGUES, L. A.; MUNIZ, T. A.; SAMARÃO, S. S.; CYRINO, A. E. Qualidade de mudas de moringa oleifera lam. cultivadas em substratos com fibra de coco verde e compostos orgânicos. Ceres, v. 63, n.4, p. 545-552, 2016. Disponível em: <http://dx.doi.org/10.1590/0034-737X201663040016>. doi: 10.1590/0034737X201663040016

RSA Indústria de Insumos Agrícolas LTDA. São Paulo, SP: Bacsol, 2014. Bula. Disponível em: <http://www.rsa.ind.br >.

SALTON, J. C.; TOMAZI, M. Sistema radicular de plantas e qualidade do solo. Dourados: Embrapa Agropecuária Oeste, 2014. 6p.(Embrapa Agropecuária Oeste: Comunicado técnico 198).

SIQUEIRA, D. P.; CARVALHO, G. C. M. W.; BARROSO, D. G.; MARCIANO, C. R. Lodo de esgoto tratado na composição de substrato para produção de mudas de Lafoensia glyptocarpa. Floresta, v. 48, n. 2, p. 277-284, 2018. Disponível em: <https://doi.org/10.5380/rf.v48i2.55795>. doi: 10.5380/rf.v48i2.55795.

SOUZA, M. C. M. R.; MENEZES, A. S.; COSTA, R. S.; AMORIM, C. V.; LACERDA, C. F.; et al. Tolerância à salinidade e qualidade de mudas de noni sob diferentes ambientes e matéria orgânica. Revista Brasileira de Agricultura Irrigada, v. 11, n. 
7, p. 2052 - 2062, 2017. Disponível em: <doi:10.7127/rbai.v11n700682>. doi:10.7127/rbai.v11n700682.

SPANIOL, J. S.; OLTRAMARI, C. E.; LOCATELLI, M.; VOLPATO, A.; CAMPIGOTTO, G. et al. Influence of probiotic on somatic cell count in milk and immune system of dairy cows. Comparative Clinical Pathology, v. 24, n. 3, p. 677681, 2014. Disponível em: <https://doi.org/10.1007/s00580-014-1966-y>. doi: 10.1007/s00580-014-1966-y

STEFFEN, G. P. K.; ANTONIOLLI, Z. I.; STEFFEN, R. B.; SCHIEDECK, G. Utilização de vermicomposto como substrato na produção de mudas de Eucalyptus grandis e Corymbia citriodora. Pesquisa Florestal Brasileira, v. 31, n. 66, p. 75-82, 2011. Disponível em: <http://agris.fao.org/agris-search/search.do? recordID=DJ2012078833>.

TOLEDO, F. H. S. F; VENTURIN, N.; CARLOS, L.; DIAS, B. A. S.; VENTURIN, R. P. et al. Composto de resíduos da fabricação de papel e celulose na produção de mudas de eucalipto. Revista Brasileira de Engenharia agrícola ambiental, v. 19, n. 7, p. 711-716, 2015. Disponível em: <http://dx.doi.org/10.1590/18071929/agriambi.v19n7p711-716>. doi: 10.1590/1807-1929/agriambi.v19n7p711-716

VIEIRA, C. R.; WEBER, O. L. S.; SCARAMUZZA, J. F. Resíduos Orgânicos como Substrato para Produção de Mudas de Guanandi. Uniciências, v. 18, n. 2, p. 91-97, 2014. Disponível em: <http://dx.doi.org/10.17921/1415-5141.2014v18n2p\%25p>. doi: $10.17921 / 1415-5141.2014 \mathrm{v} 18 \mathrm{n} 2 \mathrm{p} \% 25 \mathrm{p}$ 